\title{
Моделирование рядов среднегодовых расходов воды рек Разлив и Промысловая
}

\author{
Ю.А. Спирин $\bowtie$ \\ Балтийский Федеральный университет им. И. Канта, \\ Российская Федераиия \\ (236016, г. Калининград, ул. А. Невского, 14)
}

\begin{abstract}
Аннотация: Цель работы - произвести моделирование рядов среднегодовых расходов воды р. Разлив и р. Промысловая с использованием данных о кратковременных наблюдениях.

Mатериаль и методы. В основу моделирования лег метод «Монте-Карло». Для его реализации, были использованы данные о кратковременных гидрологических наблюдениях за исследуемыми реками, а также учтены гидрологические особенности территории.

Результаты и обсуждение. Смоделированы ряды среднегодовых расходов и на их основе построены кривые обеспеченности. Рассчитаны средние многолетние расходы, средние квадратические отклонения, коэффициенты асимметрии и вариации и их отношения, коэффициенты автокорреляции.

Заключение. Рассчитанные величины среднего многолетнего расхода, коэффициента асимметрии, вариации и автокорреляции мало отличаются от заданных величин (менее чем на 10 процентов). Наибольшее отличие получено у параметров трехпараметрического гамма-распределения $\alpha$ и $b$ : $-55,10 \%$ и -33,96 \%. Графики теоретических и рассчитанных кривых обеспеченности среднегодовых расходов практически не различаются, следовательно, сгенерированный ряд имеет заданное распределение. Полученные расчетные величины имеют высокую степень доверия, поскольку в основу теоретических характеристик легли как данные кратковременных измерений непосредственно на исследуемых водных объектах, так и полученные на базе рек аналогов территориальные гидрологические особенности. Одной из таких особенностей можно отметить хорошую синхронность колебаний речного стока среди водотоков в районе исследования. Полученные данные могут сыграть важную роль в планировании их использования, а также в различных проектных мероприятиях по развитию водопользования.
\end{abstract}

Ключевые слова: генерация гидрологических рядов, моделирование речного стока, гамма-распределение, псевдослучайные числа, гидрометрические наблюдения, река Разлив, река Промысловая.

Для цитирования: Спирин Ю.А. Моделирование рядов среднегодовых расходов воды рек Разлив и Промысловая // Вестник Воронежского государственного университета. Серия: География. Геоэкология, 2021, №2, с. 30-37. DOI: https://doi.org/10.17308/geo.2021.2/3444

\section{ВВЕДЕНИЕ}

Систематический многолетний гидрологический мониторинг водотоков является самым действенным способом в формировании гидрологических массивов данных. На основе собранной информации рассчитываются параметры рек и прогнозируется их поведение с целью использования в различных водохозяйственных и научно-прикладных отраслях. Отсутствие такого рода материалов делает невозможным любые виды адекватного гидростроительства и рационального использования водных ресурсов. Одним из самых значимых параметров можно выделить среднегодовой расход воды $[12,13]$ и максимальных расходов редкой повторяемости [9].

Полные и имеющие достаточную продолжительность гидрологические ряды каких-либо гидрологических характеристик являются больше исключением, чем правилом. Нередки случаи, когда за водными объектами прекращается наблюдение из-за отсутствия финансирования, а иногда данные о наблюдениях и вовсе теряются. Часто отсутствие гидрологической информации связано с тем, что за водным объектом не было организова-

(C) Спирин Ю. А., 2021

$\triangle$ Спирин Юрий Александрович, e-mail: spirin1234567890@rambler.ru

Контент доступен под лицензией Creative Commons Attribution 4.0 License. 
но наблюдений. Если сформированный на основе наблюдений гидрологический ряд не имеет достаточную продолжительность или он имеет пропуски, которые требуется заполнить, то прибегают к их восстановлению. Существуют различные методы моделирования гидрологического ряда.

В статье рассмотрены два водотока - река Разлив и река Промысловая. Эти водные объекты располагаются на территории осушаемого польдерного массива и являются поверхностными источниками водоснабжения поселка Мысовка и поселка Причалы, расположенных в устьевых частях рек. За водотоками не ведётся постоянный и отлаженный гидромониторинг, несмотря на то что для подобного рода пользования он необходим.

Цель работы - произвести моделирование рядов среднегодовых расходов воды рек Разлив и Промысловая с использованием данных о кратковременных наблюдениях.

\section{МАТЕРИАЛЫ И МЕТОДЫ}

Моделирование среднегодовых расходов воды рек Разлив и Промысловая будет базироваться на методе «Монте-Карло» [6, 7, 8, 10, 11, 14, 15], в рамках которого необходимо задать четыре величины: теоретический среднегодовой расход (Qst, м³/c), теоретические коэффициенты вариации, асимметрии и автокорреляции (Cvt, Cst и rt).

Информацию для получения теоретических среднегодовых расходов предоставил филиал ФГБУ «Балттехмордирекция» Калининградский с разрешения заказчика услуг СПК «Рыболовецкий колхоз «Рыбак Балтики». Расходы воды измерялись в рамках гидрохимических исследований в устьевых частях рек. Данные о мгновенных измерениях расходов воды в реке Разлив - п. Мысовка и реке Промысловая - П. Причалы были получены за период с 23.04.2013 по 27.11.2018 год (рис. 1).

Отсутствие данных о гидрометрических измерениях или их дефицит для проведения гидрометрических расчётов требует использовать метод «Восстановления гидрологического ряда по ряду аналогу» ${ }^{1}$. Однако указанный метод имеет некоторые ограничения к полученной во время наблюдений гидрологической информации. В на-

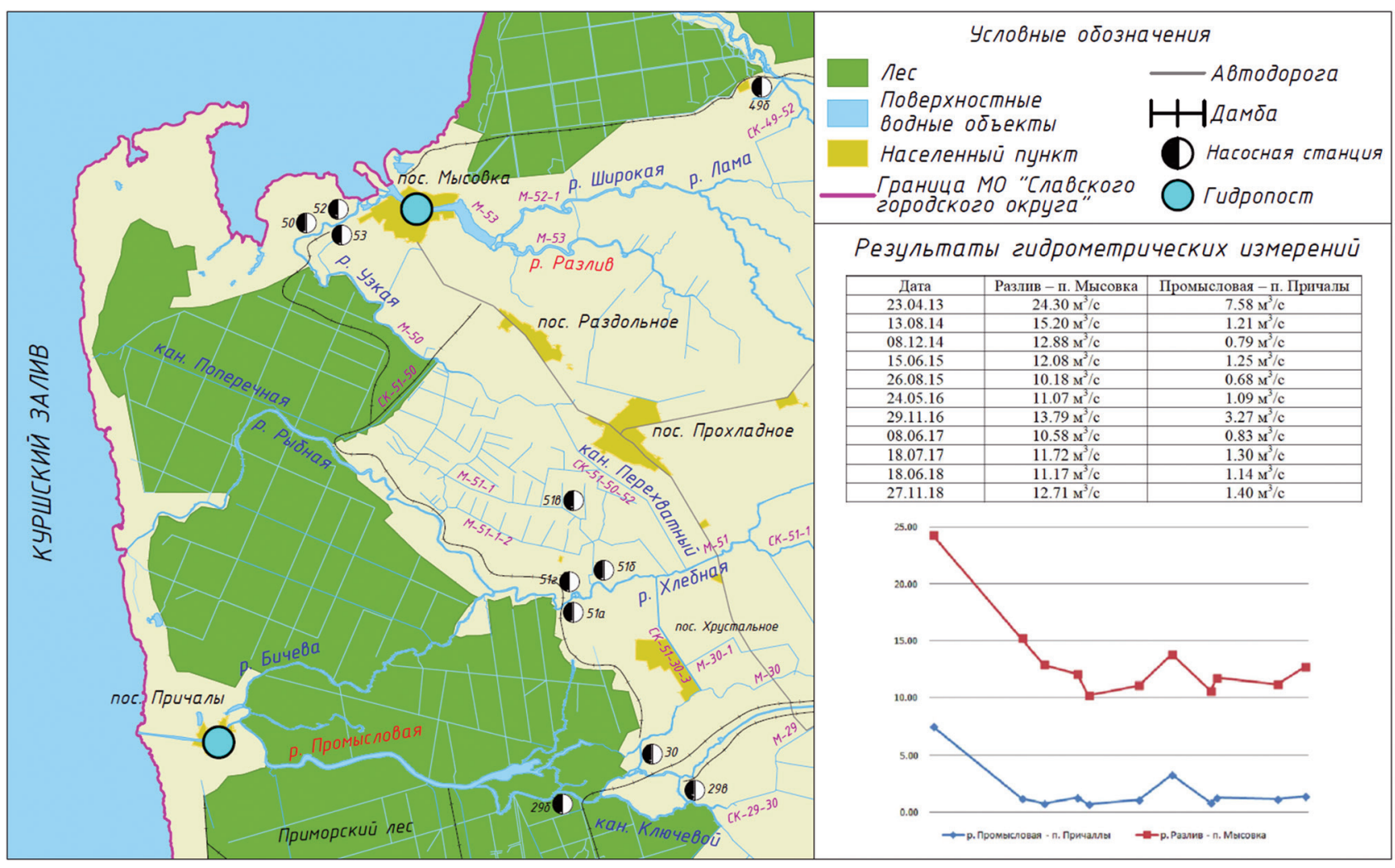

Puc. 1. Точки мониторинга и результаты гидрометрических измерений р. Разлив - п. Мысовка и р. Промысловая - П. Причалы

[Fig. 1. Monitoring points and results of hydrometric measurements of the Razliv River - p. Mysovka and the Promyslovaya River - p. Prichaly]

${ }^{1}$ Определение основных расчетных гидрологических характеристик. СП 33-101-2003: одобрен для применения в качестве нормативного документа постановлением Госстроя России № 218 от 26 декабря 2003 г. [Электронный реcypc] URL: http://docs. cntd.ru/ (дата обращения: 22.06.20).

Вестник ВГУ, Серия: География. Геоэкология, 2021, № 2, 30-37 
шем случае для использования этого метода имеющаяся выборка является не репрезентативной, так как внутри каждого отдельного года измерения не систематические и имеют недостаточную продолжительность. Не смотря на то, что данные не репрезентативны для их использования более чем достаточно, чтобы они выступили исходной опорой для формирования теоретических среднегодовых расходов при восстановлении гидрологического ряда. Наличие и осреднение материалов о кратковременных наблюдениях значительно повысит точность дополненного ряда.

Во время подбора Cvt, Cst и rt следует учитывать территориальные гидрологические особенности, сформированные реками наиболее близко отвечающие требованиям гидрологического подобия по отношению к исследуемым водотокам [6, $7,8,10,11]$. В нашем регионе три реки, которые отвечают этим принципам: река Злая, река Оса и река Немонинка. За ними велись длительные гидрологические наблюдения.

Гидрологические ряды среднегодовых расходов воды были составлены по данным «Гидрологических ежегодников» $[1,2,3,4]$ - река Злая с 1961 по 1986 год; река Оса с 1962 по 1972 год; река Немонинка с 1962 по 1986 год. Гидрологи- ческие ежегодники были предоставлены: «Государственным гидрологическим институтом»; «Институтом водных проблем РАН»; «Калининградской областной научной библиотекой». Информация о среднегодовых расходах воды реки Злая за периоды с 1990 по 1991 год и с 1993 по 2004 год получена из отчетов о «Ежегодных данных о режиме и ресурсах поверхностных вод суши» [5] и «Государственного гидрологического института». Дополнительно из «Автоматизированной системы государственного мониторинга водных объектов» были заимствованы гидрологические данные за период с 2008 по 2017 год для реки Злая. Поскольку в наблюдениях отсутствовали измерения за некоторые годы, а также часть полученных рядов имела недостаточную продолжительность, то они нами были восстановлены. На основе полученных рядов вычислены коэффициенты вариации, асимметрии и автокорреляции для рек подобия - Злая, Оса и Немонинка, чтобы их усредненный вариант использовать для подбора Cvt, Cst и rt для рек Разлив и Промысловая.

В итоге нами получены следующие исходные характеристики для выполнения поставленной цели (таблица 1).

Основные исходные теоретические характеристики для моделирования

Таблий 1 гидрологических рядов среднегодовых расходов

[Table 1. Basic initial theoretical characteristics for modeling hydrological series of average annual discharges]

\begin{tabular}{|c|c|c|c|c|}
\hline Наименование рек / River name & Qst & Cvt & Cst & rt \\
\cline { 1 - 2 } Разлив / Razliv & 13,24 & 0,45 & 0,56 & 0,21 \\
\hline Промысловая / Promyslovaya & 1,87 & & 0,56 \\
\hline
\end{tabular}

\section{РЕЗУЛЬТАТЫ И ОБСУЖДЕНИЕ}

Все дальнейшие расчёты производились в среде MathCAD. Для более лаконичного рассуждения некоторые формулы будут представлены его операторами.

Вначале запишем плотность трехпараметрического гамма-распределения для модульных коэффициентов k и зададим начальное приближение его параметров:

$$
\begin{aligned}
& f(k, \alpha, b)=\frac{1}{|b| \Gamma(\alpha)} \frac{\Gamma(\alpha+b)}{\Gamma(\alpha)} x^{\frac{a}{b}} x^{\frac{\alpha}{b} 1} \\
& \exp \frac{\Gamma(\alpha+b)}{\Gamma(\alpha)} x^{\frac{1}{b}} \text { nрu } \alpha=5 u b=5
\end{aligned}
$$

Параметры гамма-распределения $\alpha$ и b найдем из системы интегральных уравнений по заданным характеристикам Cvt и Cst с использованием операторов Given и Find:

Given

$$
\begin{gathered}
C v t=\sqrt{\int_{0}^{\infty}(k-1)^{2} \cdot f(k, \alpha, b) d k} \\
C s t=\frac{1}{C v t^{3}} \cdot \int_{0}^{\infty}(k-1)^{3} \cdot f(k, \alpha, b) d k \\
a b=\operatorname{Fimd}(\alpha, b)
\end{gathered}
$$

Полученные параметры запишем в таблицу 2. 
Параметры трехпараметрического гамма-распределения

[Table 2. Parameters of the three-parameter gamma distribution]

\begin{tabular}{|c|c|c|}
\hline Наименование реки / River name & ab1 & ab2 \\
\hline Разлив / Razliv & 1,47 & 0,53 \\
\hline Промысловая / Promyslovaya & & \multirow{2}{*}{. } \\
\hline
\end{tabular}

Теоретическая плотность и функция трехпараметрического гамма-распределения выглядит следующим образом:

$$
\begin{aligned}
& f(k)=f 1(k, \alpha, b) \\
& F(k)=\int_{0}^{k} f(t) d t
\end{aligned}
$$

Генерируем $\mathrm{n}$ псевдослучайных чисел, равномерно распределенных на отрезке $[0 ; 1]$ при помощи оператора runif:

$$
\xi=\operatorname{runif}(n, 0,1),
$$

где $\mathrm{n}=57$ - количество принятых членов ряда.
Расчёт случайных чисел с гамма-распределением проводим в последовательности, показанной на рисунке 2. Для этого используем оператор root:

$$
\phi_{i}=\operatorname{root}\left(F(u)-\xi_{i}, u\right),
$$

где $\mathrm{u}=1$ - начальное приближение (граница поиска).

Ряд модульных коэффициентов с гамма-распределением и внутренними связями рассчитываем по формулам:

$$
\begin{gathered}
k_{i}=\phi_{i} \quad k_{j}=1+r t \cdot\left(k_{j-1}-1\right)+\left(\phi_{j}-1\right) \cdot \sqrt{1-r t^{2}} \\
i=1 . . n \quad j=2 . . n
\end{gathered}
$$

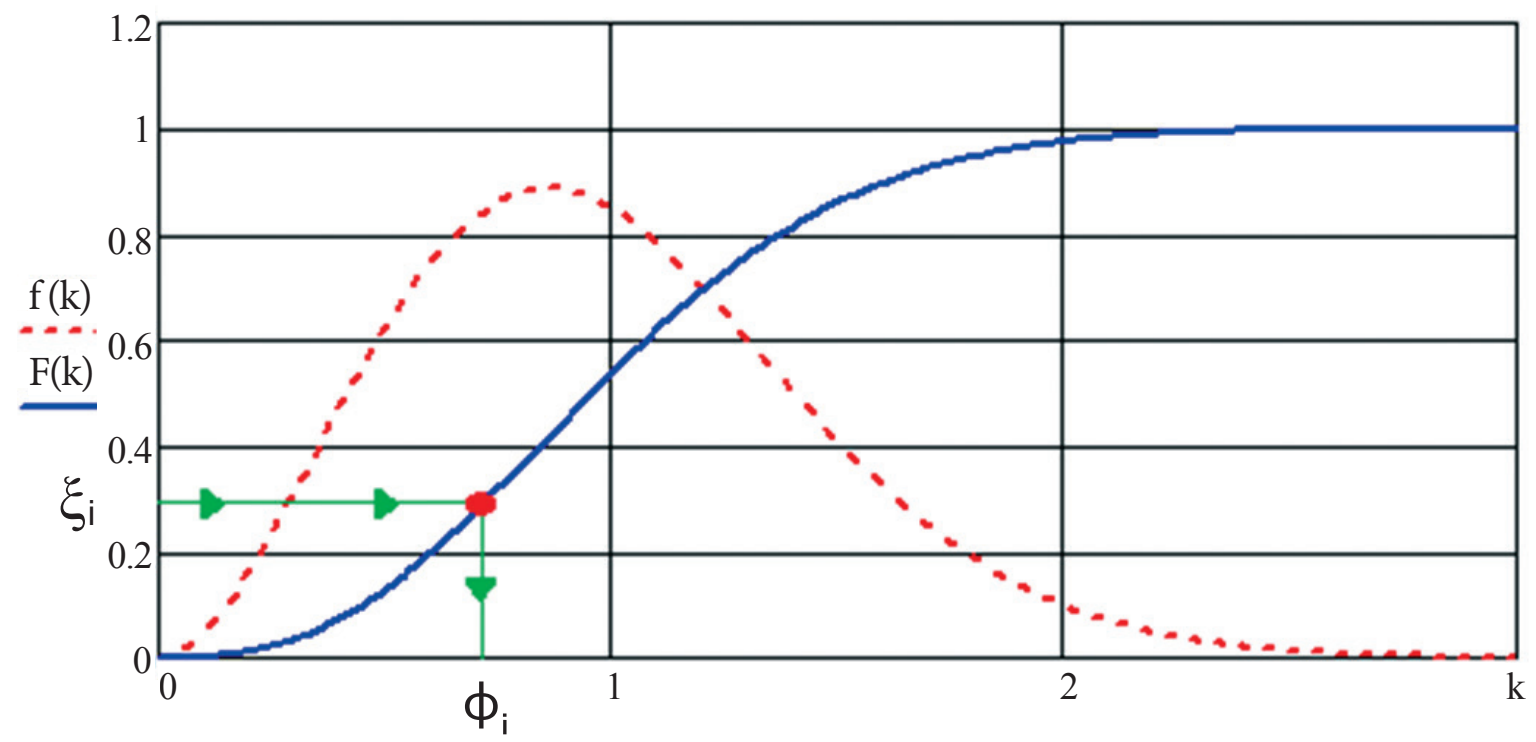

Puc. 2. Теоретическая плотность и функция трехпараметрического гамма-распределения.

Схема вычисления чисел с заданным распределением

[Fig. 2. Theoretical density and three-parameter gamma distribution function. Scheme for calculating numbers with a given distribution]

Соответствующий ряд среднегодовых расходов получим следующим образом:

$$
Q_{i}=k_{i} \cdot Q s t
$$

Рассчитаем по формулам ${ }^{2}$ и внесем в таблицу 3 числовые характеристики сгенерированных рядов такие как: средний многолетний расход $\left(\mathrm{Qs}, \mathrm{m}^{3} / \mathrm{c}\right)$; среднее квадратическое отклонение $(\sigma$, $\left.\mathrm{M}^{3} / \mathrm{c}\right)$; коэффициенты асимметрии и вариации, и их отношения (Cs, Cv и Csv); коэффициент автокорреляции (r).

${ }^{2}$ Определение основных расчетных гидрологических характеристик. СП 33-101-2003: одобрен для применения в качестве нормативного документа постановлением Госстроя России № 218 от 26 декабря 2003 г. [Электронный pecypc] URL: http://docs. cntd.ru/ (дата обращения: 22.06.20).

Вестник ВГУ, Серия: География. Геоэкология, 2021, № 2, 30-37 


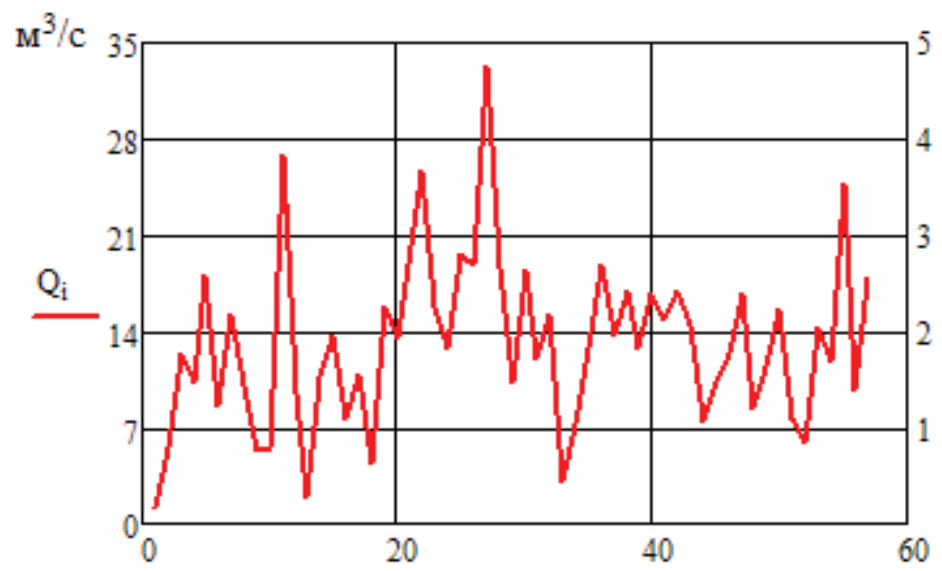

Рис. 3. Гидрологические ряды среднегодовых расходов воды р. Разлив - п. Мысовки и р. Промысловая - п. Причалы

[Fig. 3. Hydrological series of average annual water discharge in the Razliv River - p. Mysovka and the Promyslovaya River - p. Prichaly]

Таблииа 3

Основные рассчитанные характеристики смоделированных гидрологических рядов среднегодовых расходов

[Table 3. Main calculated characteristics of the modeled hydrological series of average annual discharges]

\begin{tabular}{|c|c|c|c|c|c|c|}
\hline Наименование рек / River name & Qs & $\sigma$ & Cs & Cv & Cvs & r \\
\cline { 1 - 7 } Разлив / Razliv & 13,15 & 6,22 & 0,57 & 0,47 & 1,21 & \multirow{2}{*}{0,23} \\
\hline Промысловая / Promyslovaya & 1,86 & 0,88 & & & & \\
\hline
\end{tabular}

Далее вычисляется параметр $\lambda 2$ :

$$
\lambda 2=\frac{1}{n-1} \cdot \sum_{i=1}^{n} \ln \left(k_{i}\right)
$$

Параметры трехпараметрического гамма-распределения находятся методом наибольшего правдоподобия, для чего численно решается система интегральных уравнений с помощью следующих операторов:

Given

$$
\begin{gathered}
\lambda 2+\ln \left(\frac{\Gamma(\alpha+b)}{\Gamma(\alpha)}\right)-\frac{b}{\Gamma(\alpha)} \cdot\left(\int_{0}^{\infty} t^{\alpha-1} \cdot \ln (t) \cdot \exp (-t) d t\right)=0 \\
\alpha \cdot \lambda 2-\left(\frac{\Gamma(\alpha+b)}{\Gamma(\alpha)}\right)^{\frac{1}{b}} \cdot\left[\frac{1}{n v-1} \cdot \sum_{i=1}^{n v}\left[\ln \left(k_{i}\right) \cdot\left(k_{i}\right)^{\frac{1}{b}}\right]\right]+b=0 \\
A=\operatorname{Fimd}(\alpha, b)
\end{gathered}
$$

Все полученные значения запишем в таблицу 4. Таблииа 4

Параметры трехпараметрического гамма-распределения

[Table 4. Parameters of the three-parameter gamma distribution]

\begin{tabular}{|c|c|c|c|}
\hline River name & $\lambda 2$ & A1 & A2 \\
\hline Zlaya & $-0,09$ & 2,51 & 0,62 \\
\hline Osa & $-0,17$ & 0,41 & 0,25 \\
\hline Matrosovka & $-0,02$ & 11,96 & 0,61 \\
\hline Nemoninka & $-0,13$ & 1,93 & 0,67 \\
\hline
\end{tabular}

Теоретическая вероятность превышения (обеспеченность) среднегодового расхода выглядит следующим образом:

$P o(Q x)=100 \cdot\left(1-F\left(\frac{Q x}{Q s t}\right)\right) \quad P g(Q x)=100 \cdot\left(1-F\left(\frac{Q x}{Q s}\right)\right)(6)$

Кривые обеспеченности среднегодовых расходов представлены на рисунке 4.

Произведем сравнение заданных и рассчитанных по ряду характеристик и параметров (таблица 5).

\section{ЗАКЛЮЧЕНИЕ}

В статье был сгенерирован гидрологический ряд среднегодовых расходов воды для реки Разлив - п. Мысовки и реки Промысловая - П. Причалы продолжительностью 57 лет. На основе полученных рядов были построены кривые обеспеченности среднегодовых расходов и рассчитаны основные гидрологические характеристики.

Рассчитанные величины среднего многолетнего расхода, коэффициента асимметрии, вариации и 


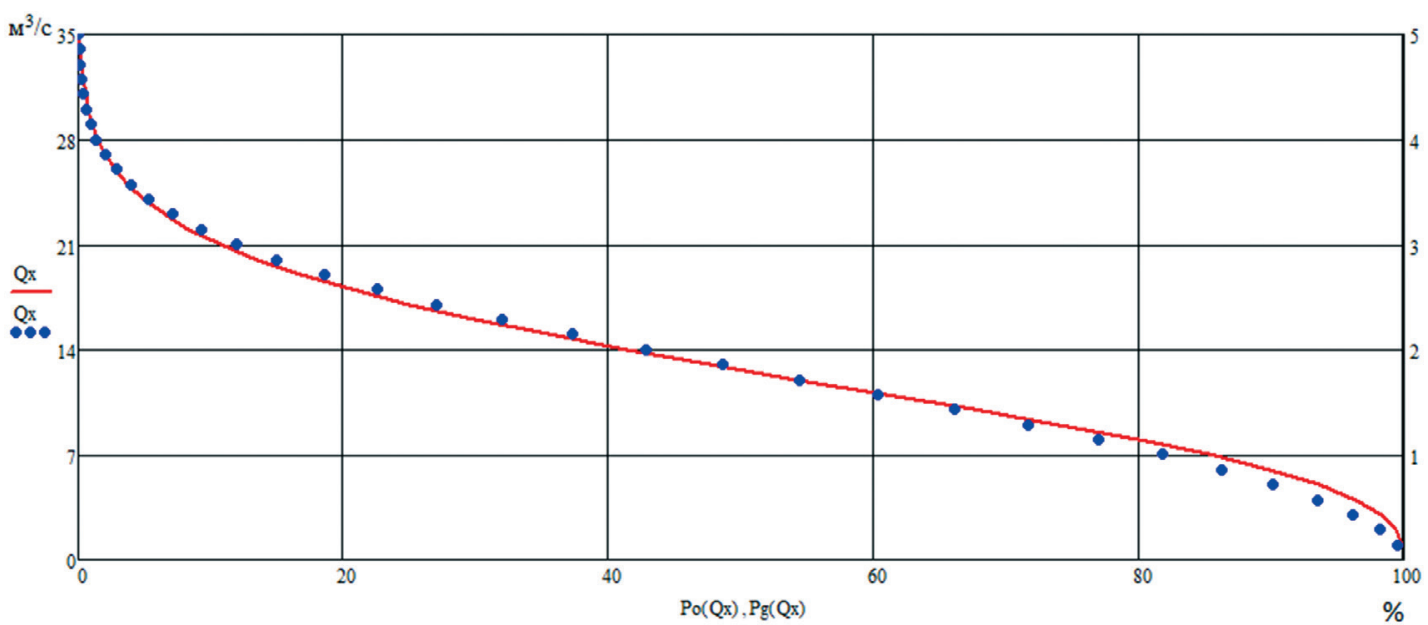

Рис. 4. Эмпирические (точки) и теоретические кривые обеспеченности среднегодовых расходов p. Разлив - п. Мысовки и р. Промысловая - п. Причалы: сплошная линия - по исходным данным, пунктирная линия (точки) - по сгенерированному ряду

[Fig. 4. Empirical (points) and theoretical curves of average annual of discharge

in the Razliv River - p. Mysovka and the Promyslovaya River - p. Prichaly: solid line - according to the original data, dashed line (dots) - according to the generated series]

Таблица 5

Сравнение заданных и рассчитанных по ряду характеристик и параметров

[Table 5. Comparison of specified and calculated for a number of characteristics and parameters]

\begin{tabular}{|c|c|c|c|c|}
\hline \multirow{2}{*}{$\begin{array}{c}\text { Характеристика, } \\
\text { параметр / } \\
\text { Feature, parameter }\end{array}$} & \multicolumn{2}{|c|}{ Величина / Value } & \multicolumn{2}{|c|}{ Отклонение / Deviation } \\
\hline & $\begin{array}{c}\text { теоретическая / } \\
\text { theoretical }\end{array}$ & $\begin{array}{c}\text { рассчитанная / } \\
\text { calculated }\end{array}$ & $\begin{array}{c}\text { абсолютное / } \\
\text { absolute }\end{array}$ & $\begin{array}{c}\text { относительное / } \\
\text { relative }\end{array}$ \\
\hline Qs $1, \mathrm{M}^{3} / \mathrm{c}$ & 13,24 & 13,15 & $-0,09$ & $-0,68 \%$ \\
\hline Qs2, $\mathrm{M}^{3} / \mathrm{c}$ & 1,87 & 1,86 & $-0,01$ & $-0,53 \%$ \\
\hline $\mathrm{Cs}$ & 0,56 & 0,57 & 0,01 & $1,79 \%$ \\
\hline $\mathrm{Cv}$ & 0,45 & 0,47 & 0,02 & $4,44 \%$ \\
\hline Csv & 1,24 & 1,21 & $-0,03$ & $-2,55 \%$ \\
\hline $\mathrm{r}$ & 0,21 & 0,23 & 0,02 & $9,52 \%$ \\
\hline$\alpha$ & 1,47 & 0,66 & $-0,81$ & $-55,10 \%$ \\
\hline $\mathrm{b}$ & 0,53 & 0,35 & $-0,18$ & $-33,96 \%$ \\
\hline
\end{tabular}

автокорреляции мало отличаются от заданных величин (менее чем на 10 процентов). Наибольшее отличие получено у параметров трехпараметрического гамма-распределения $\alpha$ и b: $-55.10 \%$ и -33.96 $\%$. Но по рисунку 4 видно, что графики теоретических и рассчитанных кривых обеспеченности среднегодовых расходов практически не различаются, следовательно, сгенерированный ряд имеет заданное распределение. Рассчитанные величины имеют высокую степень доверия, поскольку в основу теоретических характеристик легли как данные кратковременных измерений непосредственно на исследуемых водных объектах, так и полученные на базе рек аналогов территориальные гидрологические особенности. Одной из таких особенностей можно отметить хорошую синхронность колебаний речного стока среди водотоков в районе исследования.

Полученные данные могут сыграть важную роль в дальнейших исследованиях водотоков при планировании их использования в различных проектных мероприятиях по развитию водопользования.

\section{СПИСОК ЛИТЕРАТУРЫ}

1. Гидрологический ежегодник 1961-1964 г. m. 1. выл. 5, 6. Бассейны рек Немана, Преголи и Висль. Ленинград: Гидрометеоиздат, 1963-1966 г.

2. Гидрологический ежегодник 1965-1980 2. m. 1. выл. 5, 6. Бассейны рек Нямунас, Преголи и Висльл. Вильнюс, 1967-1982 г.

3. Государственный водный кадастр. Ежегодные данные о режсме и ресурсах поверхностных вод суши 1981-1984 г. ч. 1. Реки и канальл. ч. 2. Озёра и водохранилища. m. 8. выл. 4. Бассейнь рек Калининградской области. Вильнюс, 1983-1986 г.

4. Гуськов В.Г., Прошляков И.В. Моделирование взаимосвязанных стоковых рядов на примере частных водосборов бассейна реки Волги // Природообустройство, 2014, №4, с. 82-85. 
5. Джандаги Н. Моделирование рядов речного стока рек Ирана // Природообустройство, 2015, № 2, c. $59-63$.

6. Наумов В.А. Методы обработки гидрологической информации: учебник. Калининград: Издательство ФГОУ ВПО «КГТУ», 2014. $111 \mathrm{c.}$

7. Пономаренко Т.С., Рыжаков А.Н. Результаты компьютерного моделирования прохождения максимальных расходов редкой повторяемости в русле реки Альма // Вестник Воронежского государственного университета. Серия: География. Геоэкология, 2019, № 1, c. 49-54. DOI: 10.17308/geo.2019.1/2285

8. Раткович Л.Д. Моделирование взаимосвязанных гидрологических рядов // Природообустройство, 2012, № 2, c. 55-60.

9. Сикан А.В. Стохастическая модель многолетних колебаний речного стока и методика оценки ее параметров // Ученье записки РГГМУ, 2008, № 8, с. 21-27.

10. Спирин Ю.А., Ахмедова Н.Р. К вопросу о состоянии водоприемников осушительной мелиоративной сети в Калининградской области // Вестник молодёжной науки, 2014, с. 391 -393.
11. Спирин Ю.А., Зотов С.И. Проблемы геоэкологического состояния и использования поверхностных вод Калининградской области // Вестник Удмуртского университета. Серия «Биология. Науки о Земле», 2019, т. 29, № 2, c. 221-227.

12. Husin Alatas, Dyo D. Prayuda, Achmad Syafiuddin et al. Simple Model for Simulating Characteristics of River Flow Velocity in Large Scale // International Journal of Geophysics, 2015, pp. 1-8.

13. Zhenghao Zhang, Qiang Zhang, Vijay P. Singh et al. River flow modelling: comparison of performance and evaluation of uncertainty using data-driven models and conceptual hydrological model // Stochastic Environmental Research and Risk Assessment, 2018, vol. 32, pp. 26672682.

Конфликт интересов: Автор декларирует отсутствие явных и потенциальных конфликтов интересов, связанных с публикацией настоящей статьи.

Поступила в редакцию 28.09.2020 Принята к публикаичи 28.05.2021

\title{
Modeling the Series of Average Annual Water Discharge in the Razliv River and the Promyslovaya River
}

\author{
Yu.A. Spirin $\bowtie$ \\ Immanuel Kant Baltic Federal University, Russian Federation \\ (14, A. Nevsky St., Kaliningrad, 236016)
}

\begin{abstract}
The aim of this work is to simulate the series of the average annual water discharge in the Razliv River and the Promyslovaya River using data on short-term observations.

Methods. The methodology is based on the Monte Carlo method. For its implementation, data on shortterm hydrological observations of the studied rivers were used, and the hydrological features of the area were taken into account.

Results and discussions. The series of average annual expenditures were modeled and the supply curves were constructed on their basis. Average long-term expenditures, standard deviations, asymmetry and variation coefficients and their ratios, autocorrelation coefficients are calculated.

Conclusions. The calculated values of the mean multiyear flow rate, the coefficient of asymmetry, variation and autocorrelation differ little from the specified values (by less than 10 percent). The largest difference was obtained for the parameters of the three-parameter gamma distribution $\alpha$ and $b:-55.10 \%$ and $-33.96 \%$.

The graphs of theoretical and calculated curves of provision of average annual expenses practically do not differ, therefore, the generated series has a given distribution. Also, the calculated values have a high degree of confidence, since the theoretical characteristics are based on both the data of short-term measurements directly on the studied water bodies, and territorial hydrological features obtained on the basis of analogous
\end{abstract}

(C) Spirin Yu.A., 2021

$\triangle$ Yuri A. Spirin, e-mail: spirin1234567890@rambler.ru

The content is available under Creative Commons Attribution 4.0 License. 
rivers. One of these features is good synchronization of fluctuations in river flow among streams in the study area. The obtained data can play an important role in planning their use, as well as in various project activities to develop water use.

Key words: generation of hydrological series, modeling of river flow, gamma distribution, pseudorandom numbers, hydrometric observations, Razliv River, Promyslovaya River.

For citation: Spirin Yu.A. Modeling the Series of Average Annual Water Discharge in the Razliv River and the Promyslovaya River. Vestnik Voronezskogo gosudarstvennogo universiteta. Seria: Geografia. Geoekologia, 2021, no. 2, pp. 30-37. (In Russ.). DOI: https://doi.org/10.17308/geo.2021.2/3444

\section{REFERENCES}

1. Gidrologicheskiy ezhegodnik 1961-1964 g. t. 1. vyp. 5, 6. Basseyny rek Nemana, Pregoli i Visly [Hydrological yearbook 1961-1964. vol. 1. v. 5, 6. Neman's pools, Pragoli and Vistula]. Leningrad: Gidrometeoizdat, 19631966 g. (in Russ)

2. Gidrologicheskiy ezhegodnik 1965-1980 g. t. 1. vyp. 5, 6. Basseyny rek Nyamunas, Pregoli $i$ Visly [Hydrological yearbook 1965-1980. vol. 1. v. 5, 6. Namunas River Pools, Pregoli and Vistula]. Vilnius, 1967-1982 g. (in Russ)

3. Gosudarstvennyy vodnyy kadastr. Ezhegodnye dannye o rezhime i resursakh poverkhnostnykh vod sushi 19811984 g. ch. 1. Reki i kanaly. ch. 2. Ozera i vodokhranilishcha. t. 8. vyp. 4. Basseyny rek Kaliningradskoy oblasti [State Water Cadastre. Annual data on the mode and resources of surface waters 1981-1984, part. 1. Rivers and channels. part. 2. Lakes and reservoirs. vol. 8. v. 4. Pools of the Kaliningrad region rivers]. Vilnius, 1983-1986 g. (in Russ)

4. Gus'kov V.G., Proshlyakov I.V. Modelirovanie vzaimosvyazannyh stokovyh ryadov na primere chastnyh vodosborov bassejna reki Volgi [Modeling of interconnected runoff series on the example of private catchments of the Volga river basin]. Environmental Engineering, 2014, no. 4, pp. 82-85. (in Russ)

5. Dzhandagi N. Modelirovanie ryadov rechnogo stoka rek Irana [Modeling the ranks of the river runoff of the rivers of Iran]. Environmental Engineering, 2015, no. 2, pp. 59-63. (in Russ)

6. Naumov V.A. Metody obrabotki gidrologicheskoj informacii: uchebnik [Hydrological information processing methods: textbook]. Kaliningrad: Publishing house of FGOU VPO "KSTU", 2014. 111 p. (in Russ)

7. Ponomarenko T.S., Ryzhakov A.N. Rezul'taty komp'yuternogo modelirovaniya prokhozhdeniya maksimal'nykh raskhodov redkoy povtoryaemosti $\mathrm{v}$ rusle reki Al'ma [Results of computer simulation of the passage of maximum costs of rare repeatability in the direction of the Alma River]. Vestnik Voronezhskogo gosudarstven-

Спирин Юрий Александрович

Аспирант ФГАОУ ВО «Балтийский федеральный университет имени Иммануила Канта»; Институт природопользования, территориального развития и градостроительства, г. Калининград, Российская Федерация, ORCID: 0000-0003-3481-9666, e-mail: spirin1234567890@rambler.ru nogo universiteta. Seria: Geografia. Geoekologia, 2019, no. 1, pp. 49-54. (in Russ)

8. Ratkovich L.D. Modelirovanie vzaimosvyazannyh gidrologicheskih ryadov [Modeling of interconnected hydrological series]. Environmental Engineering, 2012, no. 2, pp. 55-60. (in Russ)

9. Sikan A. V. Stohasticheskaya model' mnogoletnih kolebanij rechnogo stoka i metodika ocenki ee parametrov [Stochastic model of long-term fluctuations of river runoff and a method for assessing its parameters]. Scholarly notes $R G G M U, 2008$, no. 8, pp. 21-27. (in Russ)

10. Spirin Yu.A., Ahmedova N.R. K voprosu o sostoyanii vodopriemnikov osushitel'noj meliorativnoj seti v Kaliningradskoj oblasti [On the question of the state of the drainage network in the Kaliningrad region]. Bulletin of youth science: collection of papers, 2014, pp. 391393. (in Russ).

11. Spirin Yu.A., Zotov S. I. Problemy geoekologicheskogo sostoyaniya i ispol'zovaniya poverhnostnyh vod Kaliningradskoj oblasti [Problems of the geoecological state and use of surface waters of the Kaliningrad region]. Bulletin of the Udmurt University. Series "Biology. Earth Sciences", 2019, vol. 29, no. 2, pp. 221-227. (in Russ).

12. Husin Alatas, Dyo D. Prayuda, Achmad Syafiuddin et al. Simple Model for Simulating Characteristics of River Flow Velocity in Large Scale. International Journal of Geophysics, 2015, pp. 1-8.

13. Zhenghao Zhang, Qiang Zhang, Vijay P. Singh et al. River flow modelling: comparison of performance and evaluation of uncertainty using data-driven models and conceptual hydrological model. Stochastic Environmental Research and Risk Assessment, 2018, vol. 32, pp. $2667-$ 2682.

Conflict of interests: The author declares no information of obvious and potential conflicts of interest related to the publication of this article.

Received: 28.09 .2020 Accepted: 28.05.2021

Yuri A. Spirin

Post-graduate student of Immanuel Kant Baltic Federal University; Institute of Nature Management, Territorial Development and Urban Planning, Kaliningrad, Russian Federation, 236022, ORCID: 0000-0003-3481-9666, e-mail: spirin1234567890@rambler.ru 